\title{
A Variational Principle for General Polymer Systems
}

\section{V'WARSTA'T}

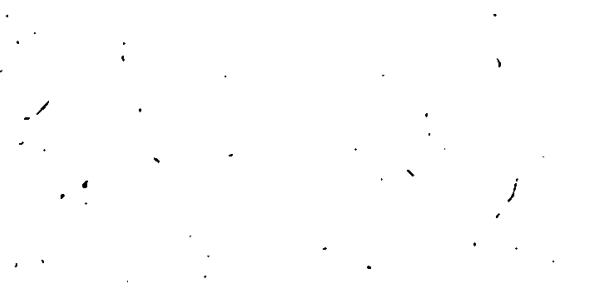

Es wird ein neues Modell für die klassische statistische Mechanik von wechselwirkenden ausgedehnten' Teilchen (Polymeren) eingeführt. Für Systeme von Polýmeren wird die Konstruktion des Konfigurationenraumes angegeben. Es folgt dẹ Nachweis für die Existenz des thermodynamischen Grenzwerțes des lokalen Drucks. Für diesen Grenzwert, den Druck des Systems, wird ein Variationsprinzip bewiesen. Abschließend wird gezeigt, daß bei gegebener Wechselwirkung die Klasse der Gleichgewichtszustände, die durch das Variationsprinzip beschrieben wird, mit der Klasse der verschiebungsinvarianten Gibbsschen Zustände zusammenfällt.

Введётся новая модель дыя классической статнстинеской механики ваапмодействующих пространственных частиц (полимеров). Для систем полимеров описывается конструкция конфигурацнонного пространства. ІІотом следует доказательство существования термодинамического. предела локального давления. Для этого предела, так называемого давления системы, доказывается вариационның принцип. Заключительно доказывается, что при данном взапмоденствии класс. равновесных состоянй, описываемый этим вариационным принципом, совпадает с классом трансляционно-инвариантных гиббсовских состояний.

$$
1
$$

A new model for classical statistical mechanics of interacting extended particles (polymers) is introduced. First, the construction of the configuration.space for polymer systems is given. Then the existence of the thermodynamic limit of the local pressure is derived. For this limit which is called the pressure of the system a variational principle is proved. Finally, for given interaction, the class of equilibrium states determined by this variational principle is proved to coincide with the class of translation invariant Gibbs states.

\section{Introduction}

Exactly soluble models are of great importance in the understanding of statistical mechanics of phase transitions. Besides the classical models, general hard core or polyner models are of interest, too [1,5-9]. In these models the particles of the system can cover more than one lattice point of the underlying lattice without penetrating each other. The most interesting examples are hard-rod models. They have been widely used to explain the existence of liquid crystals. Thus the theory of monomer-dimer systems is elaborated extensively $[1,7-9]$. In [8] the reader can find more details about the relation of these models to chemistry and physics.

Most resuits are known for models without interaction betiveen the particles; but a successful appronch to the practical models requires models with interaction. In [8] the absence of a ,phase transition is proved for translationally invariant systems of non-interacting monomers and dimers on the lattice $Z^{d}$. In [5] GRUBER and Kuvz incorporated into.their model particles that are more complicated with respect to their geometry. They showed the absence of a phase transition for non-interacting polymers and thus generalized the results by HeسMaNN and Lieb in [8]. In [9] Hercirans and Lieb sujceeded in a rigorous proof of the existence of a phase transition for monomer-dimer systems with two-body.interactions between the dimers in two dimensions. A similar result can be found in [1] for three dimensions. 
In our paper we describe a very general model for systems of polymers that can interact via many-body interactions. The underlying lattice is $\mathbf{Z}^{d}$ for $d \geqq 1$, and the polymers have the shape of finite subsets of the lattice. It is possible to have. different polymers of the same shape, but only finitely many shapes of polymers are allowed in the system. We found this model in specifying the general method for the construction of configuration spaces of classical lattice systems of RUELLE in [13].

The proof of the variational principle for the pressure of the system $[4,10]$ was a milestone -in the development of classical statistical mechanics. This result influenced not only statistical mechanics, but also ergodic theory. It was one basis for the further development of entropy theory, especially for the generalization of the variational principle for the entropy, which asserts that the topological entropy of a continuous transformation of a compact space is the supremium of the measure-theoretic entropies of regular invariant probability measures. As one generalization of entropy, RuELLE developed the concept of the pressure of a continuous transformation of a compact space in [12]. For further results in ergodic theory see $[2,3,13]$ and references therein. Some definitions and theorems that are used in our paper are summarized in the Appendix.

In the second part we introduce our model. The third part is devoted to the proof of the existence of the pressure $P(U)$ for systems of interacting polymers with interaction $U$ and to the proof of a variational principle

$$
P(U)=\sup _{\mu \in \mathcal{J}}\left|\mathscr{G}_{0}\right|^{-1}(h(\mu)-\mu(U))
$$

Herein $h(\mu)$ denotes the entropy of the invariant state $\mu, \mu(U)$ the specific energy of the system, $\left|\mathscr{G}_{0}\right|^{-1}$ the number of different shapes that polymers are allowed to have, Knowing that this variational principle is true for a particle system, the problem of phase transition reduces to the question: Does there exist more than one state $\mu$ being an equilibrium state, in the sense that it fulfils the equation

$$
P(U)=\left|\mathscr{S}_{0}\right|^{-1}(h(\dot{\mu})-\dot{\mu}(U)) ?
$$

A uniqueness theorem, i.e. a theorem, about the absence of a phase transition for some systems of interacting particles, was published in [15]. In the fourth part it is shown that the class of equilibrium states coincides with the class of translation invariant Gibbs states for the given polymer system with interaction $U$.

\section{The model}

We start with the construction of the configuration space of the polymer system. Let $\mathbf{Z}^{d}$ be the set of all $d$-tuples of integers $s=\left(s_{1}, \ldots, s_{d}\right), \mathfrak{B}_{\mathrm{f}}\left(\mathbf{Z}^{d}\right)$ the set of all nonempty finite subsets of $\mathbf{Z}^{d}$. We choose a set $\mathscr{G} \subset \mathfrak{B}_{\mathrm{f}}(\mathscr{G})$ of possible polymer shapes such that for each $s \in Z^{d}$ the set $\mathscr{L}_{s}=\{G \in \mathscr{S} \mid s \in G\}$ is non-empty and finite. Furthermore we choose for each $G \in \mathscr{E}$ a finite set $\Omega_{G}$ of possible species of polymers of the shape $G^{-}$and assume that there ${ }^{-}$is an element $O \in \Omega_{G}$ for each $G \in \mathscr{S}^{-*}$. Let ". $\bar{\Omega}_{Y}, \subset \prod\left\{\Omega_{G} \mid G \in \mathscr{L}_{s}\right\}$ be the set defined by

$$
\bar{\Omega}_{Y_{1}}=\bigcup_{G \in \mathcal{I}_{0}} \bigcup_{\omega_{G} \in \Omega_{G}}^{\cup}\left\{\left(0, \ldots, 0, \omega_{G}, 0, \ldots, 0\right)\right\} \text {. }
$$

The configuration space $\Omega$ for the polymer model is defined by

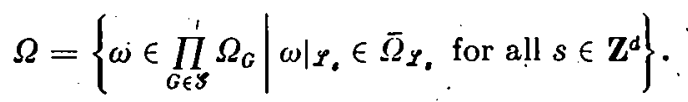


The space $\Omega$ consists of configurations $\omega$ having the property that for any point $s \in Z^{d}$ there exists at most one $G \in \mathscr{L}_{s}$ with $\left.\omega\right|_{G} \neq 0$.

So it will be convenient to interpret the configurations as arrangements of non-overlapping polymers on the lattice. The construction of the configuration space includes also the classical case of a lattice gas. To see this, choose the parameters as $\left.\mathscr{S}=\{\mid s\} \mid s \in \mathbf{Z}^{d}\right\}$ and $\Omega_{\{s\}}=\{0,1\}$. -A more abstract definition of configuration spaces with restrictions is given in [13]. Our model arises from this approach.

In the following, invariance under the group $\left(\Theta_{s}\right)_{s \in Z^{a}}$ of translations acting on the lattice'by

$$
\Theta_{s} t=t-s . \quad\left(t \in \mathbf{Z}^{d}\right)
$$

is assumed. The translate of sets $W \in \mathfrak{P}_{\mathrm{f}}\left(\mathbf{Z}^{d}\right)$ or $\mathscr{W} \subset \mathfrak{P}_{\mathrm{f}}\left(\mathbf{Z}^{d}\right)$ is denoted by $\Theta_{s} W$ or $\Theta_{s} \mathscr{W}$, respectively. The set $\mathscr{G}$ is supposed to be uniquely generated by $\left(\Theta_{s}\right)$ from 'a finite set $\mathscr{G}_{0}$ chosen in such a way that the origin of $\mathbf{Z}^{d}$ is in the lexicographic order the sinallest element in each $G \in \mathscr{G}_{0}$. Furthermore we assume that $\Omega_{G}=\Omega_{K}$ if $G=\Theta_{s} K$ for some $s \in Z^{d}$.

The configuration space $\Omega$ is to be equipped with a metric. For this let $|s|$ $=\max \left\{\left|s_{1}\right|, \ldots,\left|s_{d}\right|\right\}$ for $s=\left(s_{1}, \ldots, s_{d}\right) \in \mathbf{Z}^{d}$ and $d(G)=\min \{|s| \mid s \in G\}$ for $G \in \mathcal{G}$. Then for each $\vartheta \in(0,1)$ a metric $\underline{\varrho}_{\theta}$ on $\Omega$ is defined by

$$
\varrho_{\theta}(\omega, \eta)=\exp \left(\left(\inf _{G \in \mathcal{S}}\left\{d(G)|\omega|_{G} \neq\left.\eta\right|_{G}\right\}\right) \log \vartheta\right) .
$$

This metric is compatible with the topology induced on $\Omega \subset \Pi\left\{\Omega_{G} \mid G \in \mathscr{E}\right\}$ by the product topology on $\Pi \Omega_{G}$. On finite sets $\Omega_{G}$ the discrete topology is assumed. So $\Omega$ is seen to be compact and metrizable. On $\Omega$ the expansive group (see Appendix) . $\left(\tau_{s}\right)_{s \in \mathbf{Z}^{d}}$ of homeomorphisms $\tau_{s}$ is defined by

$$
\left.\tau_{s} \omega\right|_{G}=\left.\omega\right|_{\theta_{-,} G}, \quad\left(\omega \in \Omega, s \in \mathbf{Z}^{d}, G \in \mathscr{S}\right) .
$$

The set of all Borel probability measures on $\Omega$, the so-called states, is denoted by $\mathcal{M}$, while all translation invariant elements of $\mathscr{M}$ form the set $\mathcal{J}$.

For the proofs of the theorems we use some results of ergodic theory. They are shortly summarized in the Appendix.

\section{Thermodynamic limit and variational prineiple}

In this part we introduce interactions for systems of polymers and prove first the existence of the thermodynamic limit of the local pressure. For, this limit which is called pressure of the system the variational principle is verified. The methods of the proofs are very close to those used in [13: Chap. 3].

Given $a \in \mathbf{Z}^{+\boldsymbol{d}}=\left\{s \in \mathbf{Z}^{d} \mid s_{i} \geqq 0\right.$ for all $\left.i\right\}$, we write

$$
V(a)=\left\{s \in \mathbf{Z}^{\alpha}|| s_{i} \mid \leqq a_{i} \text { for all } i\right\}, \quad \dot{v}(a)=\{G \in \mathscr{S} \mid G \cap V(a) \neq \emptyset\} .
$$

The cardinality of sets $V \in \mathfrak{P}_{\mathrm{f}}\left(\mathbf{Z}^{d}\right)$ or $V \subset \mathfrak{P}_{\mathrm{f}}\left(\mathbf{Z}^{d}\right)$ is denoted by $|V|$ or $|\vartheta|$, respectively, while [V] denotes

$$
[V]=\cup\left\{\Theta_{s} V \mid s \in \mathbf{Z}^{d}\right\}
$$

For $G \in \mathscr{S}_{0}$ it is possible to introduce an order relation in the set $[\{G\}]$ in the follow-

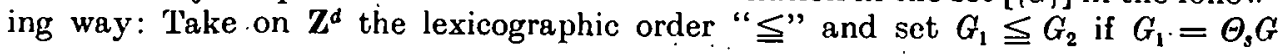


and $\dot{G}_{2}=\Theta_{t} G$ with 's $\leqq t$. The set of non-empty finite subsets of $\mathscr{G}$ is denoted by $\mathfrak{P}_{\mathrm{f}}(\mathscr{S})$.

An interaction is a map $U: \mathfrak{P}_{\mathrm{f}}(\mathscr{E}) \times \Omega \rightarrow \mathbf{R}$ such that, for fixed $v \in \mathfrak{P}_{\mathrm{f}}(\mathscr{E})$. the value $U(\nu, \omega)$ depends only on $\omega \mid v$. An interaction $U$ is translation invariant if

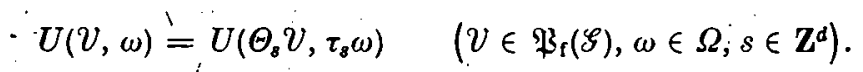

The set $(\Re,\|\cdot\|)$ of translation invariant interactions $U$ with

$$
\|U\|=\sum_{G \in \mathcal{S}_{0}} \sum_{\substack{\mathcal{W}, \mathcal{W},(\zeta) \\ \delta \in V}}|V \cap[\{G\}]|^{-1} \sup _{\omega \in \Omega}|U(V, \omega)|<\infty,
$$

is a Banach space. The same is true for the set $\subseteq \subset \Re$ with the norm

$$
\|U\|=\sum_{G \in \mathcal{S}_{0}} \sum_{\substack{v \in \mathfrak{B}_{f}(\delta) \\ \mathcal{S} \in \mathcal{V}}} \sup _{\omega \in \Omega}|U(V, \omega)| \ldots
$$

A translation invariant interaction $U$ is of finite range, $U \in \Re_{0}$, if there exists a set $\mathscr{D} \in \mathfrak{B}_{\mathrm{f}}(\mathscr{G})$ with $D \cap \mathscr{E}_{0} \neq \emptyset$ such that $U(\mathcal{V},) \neq$.0 implies the existence of an $\dot{s} \in \mathbf{Z}^{d}$ with $\Theta_{s} V \subset \mathcal{D}$.

For $V \in \mathfrak{B}_{\mathrm{r}}(\mathscr{G})$ the projection of $\Omega$ into the set $\Pi\left\{\Omega_{G} \mid G \in V\right\}$ is denoted by $X_{v} \Omega$.

For $V \subset \mathscr{G}$ finite and $U \in \Re$ we define the partition function $Z_{V}(U)$ by

$$
Z_{v}(U)=\sum_{\omega \in X_{V} \Omega} \exp \left(-\sum_{y \subset v} U\left(\mathcal{Y}, \omega^{*}\right)\right)
$$

where $\omega^{*}$ is an arbitrary configuration with $\omega^{*} \mid v=\omega$. By the definition of an interaction the partition function does not depend on the special choice of $\omega^{*}$.

The local pressure is defined by

$$
\dot{P}_{v}(U)=\mid V^{-1} \log Z_{v}(U) \quad\left(V \in \mathfrak{P}_{\mathrm{f}}(\mathscr{S}), U \in \Re\right)
$$

Our aim is to. prove the existence of the thermodynamic limit of the local pressure, i.e. the existence of $\lim P_{v_{(a)}^{\prime \prime}}(U)$ as $a \rightarrow \infty$. For proving this, to each $U \in \Re$ we adjoin a continuous function ' $A_{U}: \Omega \rightarrow \mathbf{R}$ by.

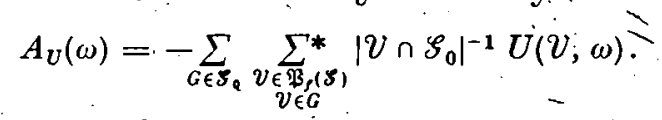

Here the second summation means that we sum up only those sets $V \in \mathfrak{B}_{\mathrm{f}}(\mathscr{S})$ for which $G$ is the $G$-greatest element in $V$ with respect to the order " $\leqq$ " in $[\{G\}]$. It is not hard to see that $U \rightarrow A_{U}$ is a linear and continuous map from $\Re$ into the set $\mathscr{E}(\Omega)$ of all continuous real functions on $\Omega$ equipped with the sup-norm. But this map is furthermore onto. We verify this in two steps:

1. For $V \in \mathfrak{B}_{f}(\mathscr{E})$ let $\mathscr{C}_{V}$ be the algebra of real functions on $\Omega$ depending only on $\omega \mid v$. It is then clear that $\left\{A_{U^{-}} \mid \cdot U \in \mathfrak{\Re}_{0}\right\}$ is a. subset of $\cup\left\{\mathscr{C}_{v} \mid . \cup \in \mathfrak{B}_{\mathrm{f}}(\mathscr{G})\right\}$, which is dense in $\mathscr{C}(\Omega)$. For the demonstration of the inverse inclusion take an arbitrary $V \in \mathfrak{B}_{\mathrm{f}}(\mathscr{S})$, an $A \in \mathscr{C}_{V}$ and an $a \in \mathbf{Z}^{+d}$ such that $V(a) \supset V$. Adjoin to $A$ the interaction $U \in \Re_{0}$ by

$$
\begin{aligned}
& U(V(a), \omega)=-A\left(\tau_{a} \omega\right) . \quad(\omega \in \Omega), \\
& U(V, \omega)=-A\left(\tau_{a-s} \omega\right) \quad \text { if, } \quad v=\Theta_{s} v(a) \quad(\omega \in \Omega), \\
& U(V, \omega)=0: \text { if } \quad v \notin[V(a)] . \quad(\omega \in \Omega) .
\end{aligned}
$$


A short calculation shows that $A_{U}=A$. Moreover, the equality $\|U\|=\left|\mathscr{S}_{0}\right|\|\dot{A}\|$ holds.

2. Let $A \in \mathscr{C}(\Omega)$. Because of the continuity of $A$ one can choose an increasing sequence $\left(V_{n}\right) \subset \mathfrak{P}_{\mathrm{f}}(\mathscr{S})$ such that

$$
\cdot \sup \left\{|A(\omega)-A(\eta)| \mid \dot{\omega}, \eta \in \Omega \text { with } \omega\left|v_{n}=\eta\right| v_{n}\right\}<\frac{1}{2^{n}}
$$

For $\omega \in \Omega$ and $n \in \mathrm{N}$ we set

$$
B_{n}(\omega)=A\left(\omega \mid v_{n}^{\prime} 0\right) \text { and } A_{1}=B_{1}, \quad A_{n}=\dot{B}_{n}-\dot{B}_{n-1},
$$

where $\omega \mid v_{n} 0$ is the configuration $\eta$ with $\eta\left|v_{n}=\omega\right| v_{n}$ and $\eta \mid s i v_{n} \equiv 0$. To the functions $A_{n} \in \mathscr{C}_{V_{n}}$ interactions $U_{n} \in \Re_{0}$ are adjoined as described in Step 1 , to the function $A$ the interaction $U=\sum U_{n}$. This procedure yields

$$
\begin{aligned}
& A_{U}(\omega)=-\sum_{G \in \mathcal{S}_{0}} \sum_{\substack{V \in \mathfrak{B}_{f}(\zeta) \\
G \in \mathcal{V}}}^{*}\left|V_{, 1} \cap \mathscr{S}_{0}\right|^{-1}\left(\sum_{n} U_{n}(\nu, \omega)\right) \\
& =\sum_{n} A_{n}(\omega)=A(\omega) \quad(\omega \in \Omega) .
\end{aligned}
$$

'The next inequality shows that $\bar{U}$ is indeed an elemient of $\mathfrak{R}$ :

$$
\|U\|=\left\|\sum_{n} U_{n}\right\| \leqq \sum_{n}\left\|U_{n}\right\|=\sum_{n}\left|\mathscr{G}_{0}\right|\left\|A_{n}\right\|<\infty
$$

The partition functions in the finite volumes $V \in \mathfrak{B}_{\mathfrak{f}}\left(Z^{d}\right)$ for continuous functions $A$ and expansiveness constants $\varepsilon>0$ are defined (see Appendix) by

$$
Z_{V}(A, \varepsilon)=\sup \left\{\sum_{\omega \in \Lambda} \exp \left(\sum_{s \in V} A\left(\tau_{s} \omega\right)\right) \mid A \text { is }(V, \varepsilon) \text {-separated }\right\}
$$

We mentioned above that the group $\left(\tau_{s}\right)$ is expansive. This is true especially for the expansiveness constant $\varepsilon=\vartheta$ (see (1)). It is not hard to show that for $a \in \mathbf{Z}^{+d}$. the $(V(a), \vartheta)$-separated subsets $\Lambda \subset \Omega$ have the property

$$
\omega, \eta \in \Lambda \text { and } \omega \neq \eta \Rightarrow \omega\left|v_{(a)} \neq \eta\right| v_{(a)} \text {. }
$$

So we can write the partition function in $V(a)$ for $A$ and $\varepsilon=\vartheta$ in the form

$$
Z_{V(a)}(A)=\sum_{\omega \in X_{V(a)} \Omega} \exp \left(\sum_{s \in V(a)} A\left(\tau_{s} \omega^{*}\right)\right)
$$

where the configurations $\omega^{*}$ with $\omega^{*} \mid v_{(a)}=\omega$ form that $(V(a), \vartheta)$-separated set $A$ that gives the supremum in $Z_{V(a)}(A, \vartheta)$. It is known (see Appendix) that the thermodynamic limit

$$
\lim _{a \rightarrow \infty}|V(a)|^{-1} \log Z_{\nu(a)}(A)=P(A)
$$

exists for all $A \in \mathscr{C}(\Omega)$, and because of $|\mathcal{V}(a)| /|V(a)| \rightarrow\left|\mathscr{G}_{0}\right|$ as ' $a \rightarrow \infty$ there exists also the limit

$$
\lim _{a \rightarrow \infty}\left|V_{i}(a)\right|^{-1} \log Z_{v_{(a)}}(A)=\left|\mathscr{S}_{0}\right|^{-1} P(A)
$$

Theorem 1: For each interaction $U . \in \mathfrak{R}$ the thermodynamic limit

$$
\lim _{a \rightarrow \infty}|V(a)|^{-1} \log Z_{v_{(a)}}(U) \stackrel{=}{=} \lim _{a \rightarrow \infty} P_{\dot{v}_{(a)}}(U)=\left|\mathscr{S}_{0}\right|^{-1} P\left(A_{0}\right)
$$

exists and is called the pressure $P(U)$ of $U$. 
Proof: 1. Let $U$ be of finite range and let $\mathcal{D}$ be the set determining the range of $U$. If $a \in \mathbf{Z}^{+d}$ is sufficiently large and ' $N(V(a))=\left|\left\{s \in V(a) . \mid \Theta_{s} D \leftarrow V(a)\right\}\right|$, then for all $\omega \in \Omega$

$$
\begin{aligned}
& \left|\sum_{y \subset V(a)} U(\mathcal{Y}, \dot{\omega})+\sum_{s \in V(a)} A_{\delta}\left(\tau_{s} \omega\right)\right| \\
& =\left.\left|\sum_{G \in \mathcal{S}_{0}} \sum_{\substack{y \in \mathfrak{P}_{\mathrm{f}}(\delta) \\
G \in \mathcal{Y}}}\right| \mathcal{Y} \cap \mathscr{S}_{0}\right|^{-1}\left(\sum_{\substack{\delta \in Z^{d} \\
\theta_{s} \mathcal{Y} \supset v(a)}} U\left(\mathcal{Y}, \tau_{s} \omega\right)-\sum_{s \in V(a)} U\left(\mathcal{Y}, \tau_{s} \omega\right)\right) \mid
\end{aligned}
$$

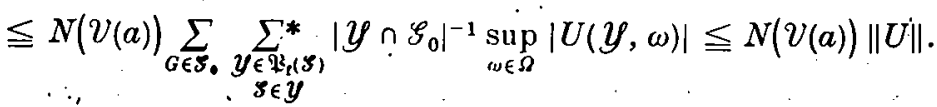

Bećause of $N(V(a)) /|V(a)| \rightarrow 0$ as $a \rightarrow \infty$, one can calculate

$$
\begin{aligned}
& \lim _{a \rightarrow \infty}\left|V_{(}(a)\right|^{-1}\left|\log Z_{V_{(a)}}(U)-\log Z_{V_{(a)}}\left(A_{U}\right)\right| \\
& =\lim _{a \rightarrow \infty}|V(a)|^{-1}\left|\log \frac{\sum_{\omega \in X_{v_{(a)}}} \exp \left(-\sum_{y \subset v_{(a)}} U\left(Y, \omega^{*}\right)\right)}{\sum_{\omega \in X_{v_{(a)}} \Omega} \exp \left(\sum_{s \in V(a)} A\left(\tau_{s} \omega^{*}\right)\right)}\right| \\
& \leqq \lim _{a \rightarrow \infty}|V(a)|^{-1} N(V(a))\|U\|=0
\end{aligned}
$$

This proves the theorem for $U \in \Re_{0}$.

2. For an arbitrary $U \in \Re$ we take into account that for $U^{\prime} \in \mathfrak{R}, t \in \mathbf{R}$ and $a \in \mathbf{Z}^{+d}$

$$
\text { 1. }\left|\frac{d}{d t} P_{v_{(a)}}\left(U+t U^{\prime}\right)\right| \leqq\|U\||V(a+c)| /|\nu(a)|
$$

holds with $c \in \mathbf{Z}^{+d}$ defined by $c_{i}=\max \left\{\left|s_{i}-t_{i}\right| \mid s \in G^{\prime}, t \in G^{\prime \prime}\right.$ for some $\left.G^{\prime \prime}, G^{\prime \prime} \in \mathscr{E}_{0}\right\}$,. $i=1, \ldots, d$. This yields

$$
\begin{aligned}
\left|P_{v_{(a)}}(U)-P_{v_{(a)}}\left(U^{\prime}\right)\right| & \leqq \sup _{0 \leqq \imath \leqq 1}\left|\frac{d}{d t} P_{v_{(a)}}\left(U+t\left(U^{\prime}-U\right)\right)\right| \\
& \leqq\left\|U-U^{\prime}\right\||V(a+c)| /|V(a)| .
\end{aligned}
$$

Because of $|V(a+c)| /|V(a)| \rightarrow\left|\mathscr{G}_{0}\right|^{-1}$ as $a \rightarrow \infty$ and the possibility to approximate $U$ by elements $U^{\prime} \in \Re_{0}$, the last estimate ensures the existence of the thermodynamic limit of the local pressure of $U$. From the continuity of the maps $U \rightarrow A_{\dot{U}}$ and $A \rightarrow P(A)$ we deduce that this limit is equal to $\left|\mathscr{E}_{0}\right|^{-1} P\left(A_{U}\right)$

Theorem 2: For $\mu . \in$ J J and $U \in \Re$ the finite thermodynamic limit

$$
\mu(U)=\lim _{a \rightarrow \infty}|V(a)|^{-1} \int\left(\sum_{y \subset v(a)} U(y, \omega)\right) d \mu(\omega)
$$

exists. Furthermore $\mu(U)=-\int A_{\sigma}(\omega) d \mu(\omega)$.

Proof: For $U \in \Re_{0}$ the theorem follows from a multidimensional version of Birkhoff's ergodic theorem (see [14]) and inequality (2). An interaction $U \in \Re$ can again be approximated by interactions of $\mathfrak{R}_{0}$ 
In the situation of polymer systems the entropy $h(\mu)$ of a state $\mu \in \mathcal{J}$ (see Appendix) is calculated as

$$
\dot{h}(\mu)=-\lim _{a \rightarrow \infty}|V(a)|^{-1} \sum_{\omega \in X_{V(a)} \rho}\left(X_{V_{(a)}} \mu\right) \cdot(\omega) \log \left(X_{V_{(a)}} \mu\right)(\omega) .
$$

Let $\mathscr{C}^{*}$ denote the weak dual of $\mathscr{C}(\Omega)$. For $A \in \mathscr{C}(\Omega)$ we set

$$
\mathcal{J}_{A}=\left\{\mu \in \mathscr{C}^{*} \mid P(A+B) \geqq P(A)+\mu(B) \text { for all } B \in \mathscr{C}(\Omega)\right\} \text {. }
$$

The elements of $\mathcal{J}_{A}$ are called equilibrium states for $A$, those of $J_{A_{v}}$ for an interaction $U$, equilibrium states for $U$. Now we can formulate our main result.

- Variational principle: For each interaction $U \in \Re$,

and

$$
P(U)=\sup _{\mu \in \mathcal{J}}\left\{\left|\mathscr{S}_{0}\right|^{-1}(h(\mu)-\mu(U))\right\}
$$

$$
\mathcal{J}_{A_{v}}=\left\{\left.\mu \in \mathcal{J}|P(U)=| \mathscr{S}_{0}\right|^{-1}(h(\mu)-\mu(U))\right\} .
$$

The set $\mathcal{J}_{A_{U}}$ is non-empty, convex and compact.

This result follows from Theorems 1 and 2 and the next Lemma, which is due to RUELeE [13] together with the variational principle quoted in the Appendix.

Lem ma: For each $A \in \mathscr{C}(\Omega)$ the following assertions are true.

(i) $\mathscr{J}_{A}=\{\mu \in \mathcal{J} \mid P(A)=h(\mu)+\mu(A)\}$.

(ii) $\mathcal{J}_{A}$ is non-empty, convex and compact.

(iii) Let $\mathfrak{X}$ be a separable Banach space, $\varphi: \mathfrak{X} \rightarrow \mathscr{C}(\Omega)$ a continuous linear map with dense domain, and for $\Phi \in \mathfrak{X}$ define

$$
\mathfrak{J}_{\Phi}^{\prime}=\left\{F \in \mathfrak{X}^{*} \mid P \circ \varphi(\Phi+\Psi) \geqq P \circ \varphi(\Phi)+F(\Psi) \text { for all } \Psi \in \mathfrak{X}\right\} \text {. }
$$

Then $\mathcal{J}_{\Phi}^{\prime}=\left\{\sigma \circ \varphi \mid \sigma \in \mathcal{J}_{\varphi \oplus}\right\}$.

, In our special case of polymer systems one has to choose $\mathfrak{X}=\mathfrak{R}$ and $\varphi: U \rightarrow A_{U}$ and to mention that $\cup\left\{\mathscr{C}_{v} \mid \mathcal{V} \in \mathfrak{P}_{\mathrm{f}}(\mathscr{S})\right\}$ is dense in $\mathscr{C}(\Omega)$.

\section{Gibbs states}

In the set $\mathscr{A}$ of all states the class of Gibbs states for a given interaction is of great interest in statistical mechanics [11]. It is our aim to show that the equilibrium states are $\tau_{8}$-invariant

- Gibbs states and vice versa.

A state is called Gibbs state for the interaction $U$ if for each finite set $V \subset \mathscr{S}$ the conditional probability that on $v$ there is the configuration $\omega_{v} \in X_{v} \Omega$, knowing that on the set $\mathscr{G} \backslash V$ there is the configuration $\eta \mid s \backslash v$ for some $\eta \in \Omega$, is cqual to.

with

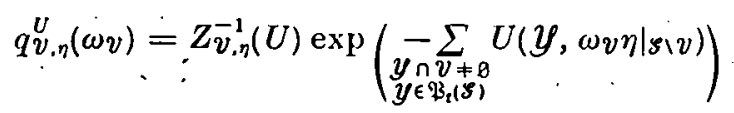

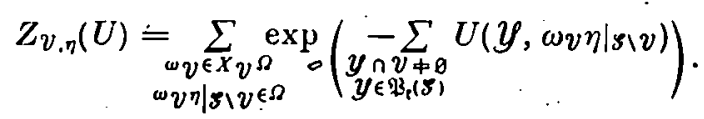

It is a known fact that the set $K_{U}$ of all Gibbs states for $U \in \Subset$ is non-empty, convex and compact (see [13]). 
Theorem 3: For each invariant polymer configuration space $\Omega$ on $\mathbf{Z}^{d}$ the class of $\tau_{s}$-invariant Gibbs states for an inleraction $U \in \mathbb{S}$ coincides with the set of equilibrium states of $U$.

The following observation is an essential step towards the proof:

Given the configuration space $\Omega$, one can find a $\bar{c} \in \mathbf{Z}^{+d}$ such that for all $a \in \mathbf{Z}^{\prime} \mathbf{Z}^{+d}$. and all $\omega, \eta \in \Omega$ there exists a $\xi \in \Omega$ with the property $\omega|v(a)=\xi| v(a)$ and $\eta \mid \xi \backslash v(a+i)$ $=\xi \mid s \backslash v(a+\bar{c})$.

Let $V=\left\{s \in \mathbf{Z}^{d} \mid\right.$ 's $\in G$ for some $\left.G \in \mathscr{S}_{0}\right\}$ and for $i=1, \ldots, d$ set $\overline{c_{i}}=$ $\max \left\{\left|s_{i}-t_{i}\right| \mid s, t \in V\right\}$. The $d$-tuple $\bar{c}=\left(\bar{c}_{1}, \ldots, \bar{c}_{d}\right)$ has the desired property. To see this take any $a \in \mathbf{Z}^{+d}, \omega, \eta \in \Omega$ and verify with the help of the definition of $\Omega$ that

$$
\xi \in \prod_{G \in \mathcal{S}} \Omega_{G} \text { with }\left.\xi\right|_{\{G\}}=\left\{\begin{array}{l}
\left.\dot{\omega}\right|_{\{G\}} \text { for } G \in V(a), \\
\left.\eta\right|_{\{G\}} \text { for } G \in \mathscr{S} \backslash V(a+\bar{c}), \\
0 \text { otherwise }
\end{array}\right.
$$

is indeed a configuration. Now one can carry out the proof of Theorem, 3 analoguously to the proof of a similar theorem of RUELLE for systems with a configuration space that is a subset of $\Omega_{0}{ }^{Z^{d}}$ for a finite set $\Omega_{0}$ (see [13: Theorem 4.2])

\section{Appendix}

We summarize here some definitions and theorems of ergodic theory which are the basis for the proofs of the Theorems 1 and 2 . More details can be found in $[2,3,12,13]$.

Let $\Omega$ be a compact space with metric $\varrho$ and $\left(\tau_{s}\right)_{s \in Z^{a}}$ a group of homeomorphisms on $\dot{\Omega}$ isomorphic to $\mathbf{Z}^{d}$.

1. Expansiveness: The group $\left(\tau_{s}\right)$ is called expansive if for an $\varepsilon>0$ and for all $\omega, \eta \in \Omega$ the implication

$$
\varrho\left(\tau_{s} \omega, \tau_{s} \eta\right) \leqq \varepsilon \text { for all } s \in \mathbf{Z}^{d} \Rightarrow \omega=\eta
$$

is true. The number $\varepsilon$ is called expansiveness constant.

2. Entropy: For $\tau_{\dot{g}}$-invariant Borel probability measures $\mu$ on $\Omega$ and finite Borel partitions $\mathfrak{A}=\left(\mathfrak{A}_{i}\right)$ of $\Omega$ we set

$$
H(\mu, \mathfrak{X})=-\sum_{i} \mu\left(\mathfrak{A}_{i}\right) \log \mu\left(\mathfrak{A}_{i}\right), \quad \mathfrak{R}^{\nabla}=\bigvee_{s \in V} \tau_{s} \mathfrak{A} \quad\left(V \in \mathfrak{P}_{(}\left(\mathbf{Z}^{\dot{d}}\right)\right) .
$$

Then $h_{\mathfrak{r}}(\mu, \mathfrak{U})=\lim _{a \rightarrow \infty}|V(a)|^{-1} H(\mu, \mathfrak{A} V(a))$ exists. The number $h(\mu)=\sup _{\mathfrak{I}} h_{\mathfrak{r}}(\mu, \mathfrak{A})$ is called the entropy of the measure $\mu$.

We write $\operatorname{diam} \mathscr{U}=\max \operatorname{diam} \mathfrak{A}_{i}$, where $\operatorname{diam} \mathfrak{A}_{i}$ is the diameter of $\mathfrak{A}_{i}$ with respect to the, metric $\varrho$.

3. Proposition: If the group $\left(\tau_{s}\right)$ is expansive with expansiveness constant $\varepsilon$, then ${ }_{--} \operatorname{diam} \mathfrak{A} \leqq \varepsilon_{-} \Rightarrow h(\mu)=h_{\mathfrak{z}}(\mu, \mathfrak{l})$.

4. Partition function: Let $V$ be a non-empty finite subset of $Z^{d}$ and $\varepsilon>Q$. A finite set $\Lambda \subset \Omega$ is called $(V, \varepsilon)$-separated if for some $s \in V$

$$
\omega, \dot{\eta} \in \Omega \text { and } \omega \neq \eta \Rightarrow g\left(\tau_{s} \omega, \tau_{s} \eta\right)>\varepsilon
$$

For $A \in \mathscr{C}(\Omega), V \in \mathfrak{B}_{\mathfrak{P}}\left(\mathbf{Z}^{d}\right)$ and $\varepsilon>0^{-}$the partition function $Z_{V}(A, \varepsilon)$ is defined by

$$
Z_{V}(A, \varepsilon)=\sup \left\{\sum_{\omega \in \Lambda} \exp \left(\sum_{s \in V} A\left(\dot{\tau}_{s} \omega\right)\right) \mid \Lambda \text { is }(V, \varepsilon) \text {-separated }\right\} \text {. }
$$


5. Pressure: If the group $\left(\tau_{s}\right)$ is expansive with expansiveness constant $\varepsilon$ and $A \in \mathscr{C}(\Omega)$, then the limit

$$
P(A)=\lim _{a \rightarrow \infty}|V(a)|^{-1} \log Z_{V(a)}(A, \varepsilon)
$$

exists independently of $\varepsilon$. The value $P(A)$ is called the pressure of the function $A$. Either $P(A)=+\infty$ for all $A \in \mathscr{C}(\Omega)$, or $P(A)<+\infty$ for all $A \in \mathscr{C}(\Omega)$. In the latter cáse the map $A \rightarrow P(A)$ is convex, increasing and continuous.

6. Variational principle: If $\mathcal{J}$ denotes the set of all $\tau_{s}$-invariant Borel probability measures on $\Omega$ and $A \in \mathscr{C}(\Omega)$, then

$$
P(A)=\sup \{h(\mu)+\mu(A) \mid \mu \in \mathcal{J}\} .
$$

In the case of finite pressure and expansive group $\left(\tau_{s}\right)$ the set

$$
J_{A}=\{\mu \in \mathcal{J} \mid P(A)=h(\mu)+\mu(A)\}
$$

is non-empty, convex and compact and coincides with the set

$$
\mathcal{J}_{A}^{\prime}=\{\mu \in \mathcal{J} \mid P(A+B) \geqq P(A)+\mu(B) \text { for all } B \in \mathscr{C}(\Omega)\} \text {. }
$$

\section{REFERENCES}

[1] ABrahaM, D. B.: Interacting dimers on the simple cubic lattice as a model for liquid crystals. J. Phys. A 13 (1980), 1051-1062.

[2] Bowen, R.: Equilibrium states and ergodic theory of Anosov diffeororphisms. Lect. Notes Math. 470 (1975).

[3] Denkker, M., Grillenberger, C., and K. Signund: Ergodic theory on compact spaces. Lect. Notes Math. $\mathbf{5} \geq 7$ (1976).

[4] Galla votti, G.', and S. Mrracle-SolÉ:Statistical mechanits of lattice systems. Comm. Math. Phys. 5 (1967), 317-323.

[5] Gruber, C., and H. Kunz: General properties of polymer systems. Comm. Math. Phys. $22(1971), 133-161$.

[6] Gruber, C., Hintermane, A., and D. Merlini: Group analysis of classical systems. Lect. Notes Phys. 60 (1977).

[7] Heilmays, O. J., and E. H. Lifb: Monomers and dimers. Phys. Rev. Letters $\mathbf{2 4}$ (1970), $1412-1414$.

[8] Hemmans, O. J., and E. H. Lieb: Theory of monomer-dimér-systems. Comm. Math. Phys. 25 (1972), 190-232.

[9] Hommane, O. J., and E. H. Lieb: Lattice models for liquid crystals. J. Stat. Phys. 20 (1979), 679-693.

[10] Ruelle, D.: A variational formulation of equilibrium statistical mechanics and the Gibbs phase rule. Comm. Math. Phys. 5 (1967), 324-329.

[11] Ruelle, D.: Statistical mechanics. New York: W. A. Benjamin Inc. 1969.

[12] RuElLE, D.: Statistical mechanics on a compact set with $\mathbf{Z}^{*}$-action satisfying expansiveness and specification. Trans. Amer. Math. Soc. 185 (1973), 237-251.

[13] Roelle, D.: Thèrmodynamic formalism. London-Amsterdam: Addison-Wesley Publ. Comp. 1978.

[14] Темпельман, А. А.: Эргодические теоремы для общих динамических систем. Труды Моск. Мат. Общества 26 (1972), 95-132.

[15] WARstat, V.: A uniqueness theorem for systems of interacting polymers. Comm. Math. Phys. 102 (1985), 47-58.

Manuskripteingang: 03. 09.1985

\section{VERFASSER :}

Dr. Volker Warstat

Sektion'Mathematik

der Martin-Luther-Univer'sität Halle - Wittenberg

Postfach

DDR - 4020 Halle 